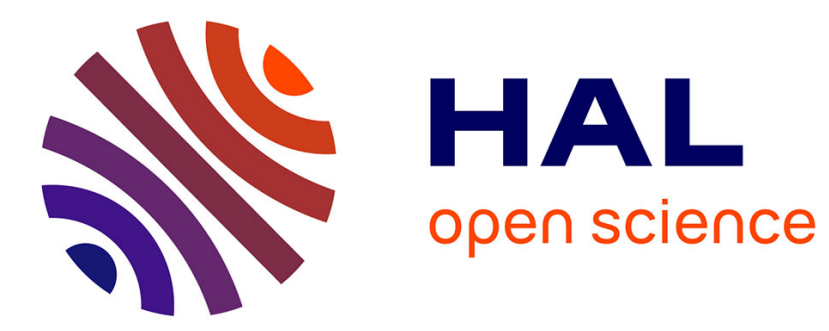

\title{
Phonons in the presence of a planar defect
}

\author{
P. Masri, L. Dobrzynski
}

\section{To cite this version:}

P. Masri, L. Dobrzynski. Phonons in the presence of a planar defect. Journal de Physique, 1975, 36

(6), pp.551-554. 10.1051/jphys:01975003606055100 . jpa-00208285

\section{HAL Id: jpa-00208285 https://hal.science/jpa-00208285}

Submitted on 1 Jan 1975

HAL is a multi-disciplinary open access archive for the deposit and dissemination of scientific research documents, whether they are published or not. The documents may come from teaching and research institutions in France or abroad, or from public or private research centers.
L'archive ouverte pluridisciplinaire HAL, est destinée au dépôt et à la diffusion de documents scientifiques de niveau recherche, publiés ou non, émanant des établissements d'enseignement et de recherche français ou étrangers, des laboratoires publics ou privés. 


\title{
PHONONS IN THE PRESENCE OF A PLANAR DEFECT
}

\author{
P. MASRI \\ Laboratoire des Mécanismes de la Croissance Cristalline $(*)$ \\ Université d'Aix-Marseille II - Centre de Luminy, 13288 Marseille, France \\ L. DOBRZYNSKI $(* *)$ \\ Institut Max-von-Laue-Paul-Langevin \\ B.P. 156, 38042 Grenoble Cedex, France \\ (Reçu le 4 décembre 1974, révisé le 20 janvier 1975, accepté le 21 février 1975)

\begin{abstract}
Résumé. - Nous montrons sur un modèle atomique et invariant par rotation que deux branches de phonons localisés dues à un défaut plan dans un cristal peuvent exister. Une de ces deux branches peut à la limite élastique rentrer dans les bandes de phonons de volume pour donner une résonance généralement bien définie.
\end{abstract}

\begin{abstract}
We report on a rotationally invariant atomic model the possibility of two distinct branches of phonons localized at a planar defect. One of these branches falls inside the bulk band in the elastic limit and transforms in a generally well defined resonance.
\end{abstract}

Crystals present planar defects (grain boundaries for example), as well as point and line defects [1]. The free surface considered as a plane defect has been extensively studied in the last few years [2]. Acoustic surface phonons are now easily generated and propagated on these surfaces; and they are even used as electronic devices [2].

The lattice vibrations in the presence of a linear or planar defect consisting of isotopic atoms has been considered by several authors [3-5]. Models of planar defects described by variations of force constants between two neighbour planes were also studied [6-8]. In all these works it was assumed that there was no coupling between atomic displacements along different crystallographic orientations $(\sigma=x, y, z)$. This unrealistic assumption does not lead to unphysical results [3] for the localized or resonant phonons as long as the defect does not admix different states, $\sigma$; this is particularly the case for purely isotopic planar defects [3-5]. However, when one assumes [6-8] that the force constants change between neighbour planes, this unrealistic assumption leads to a lack of rotational invariance in the vicinity of the planar defect. This has already been pointed out [3] in the case of a free surface, where such models were unable to show Rayleigh waves. In order to remove this difficulty, one has

(*) Associé au C.N.R.S.

(**) Present address : Laboratoire de Physique des Solides I.S.E.N., 3, rue François-Baës, 59046 Lille Cedex, France. to introduce into the equations of motion of the surface atoms, a new interaction coupling different states $\sigma$. The magnitude of this interaction is determined [3] by the rotational invariance condition. Nevertheless this was not done in the preceding studies [6-8] of non purely isotopic planar defects, which as a consequence do not give reliable results for localized phonons. We have, however, to acknowledge that all the preceding studies [3-8] of planar defects give good formal and qualitative information about quantities involving the variation in the phonon density of states. They [3-8] were even able to predict that the localized states near a planar defect will, in the acoustic region, have a dispersion relation of the form $\omega=c_{t} k+d k^{2}$ differing from the transverse bulk phonons only in the value of $d$. But because of the lack of rotational invariance of the models used [6-8], their value of $d$ cannot be expected to be correct from a physical point of view. To our knowledge only one [9] theoretical study of localized and resonant phonons near a physically realistic planar defect appeared. This work was done in the frame of elasticity theory.

We used [10] recently a simple lattice dynamical model obeying the rotational invariance condition to study the dispersion curves of surface phonons with and without an adsorbed layer and also the interface phonons at a point of high symmetry $\left(\pi / a_{0}, 0\right)$ of the two-dimensional Brillouin zone. With the same model we study here along an axis of the two-dimensional Brillouin zone, the localized and resonant phonons due to a planar defect described 
by the variation of the interaction between two neighbours planes. Our results in the long wavelength limit are compared to those of Kosevich et al. [9].

For simplicity, we consider a simple cubic monoatomic crystal with central force interactions between first $(K)$ and second nearest neighbors $\left(\frac{1}{2} K\right)$. These forces are derived from central potentials, with the assumption that the first derivatives of these potentials are negligible and set equal to zero. The inclusion of these first derivatives is important when one wants to study surface [11] or interface $(*)$ [12] superstructures. We describe schematically the planar defect by a variation of the interactions between two adjacent (001) planes; let us designate these interactions by $\left(K^{\prime}\right)$ and $\left(\frac{1}{2} K^{\prime}\right)$ respectively for the first and second nearest neighbors. This defect problem can be solved [10] by the Green's function method which enables one to calculate resonances as well as localized states. Noting that our planar defect has the symmetry of reflexion through a fictious mirror plane situated in its middle, we can block diagonalize our problem and study separately the vibrations corresponding to atomic displacements respectively symmetrical and antisymmetrical through

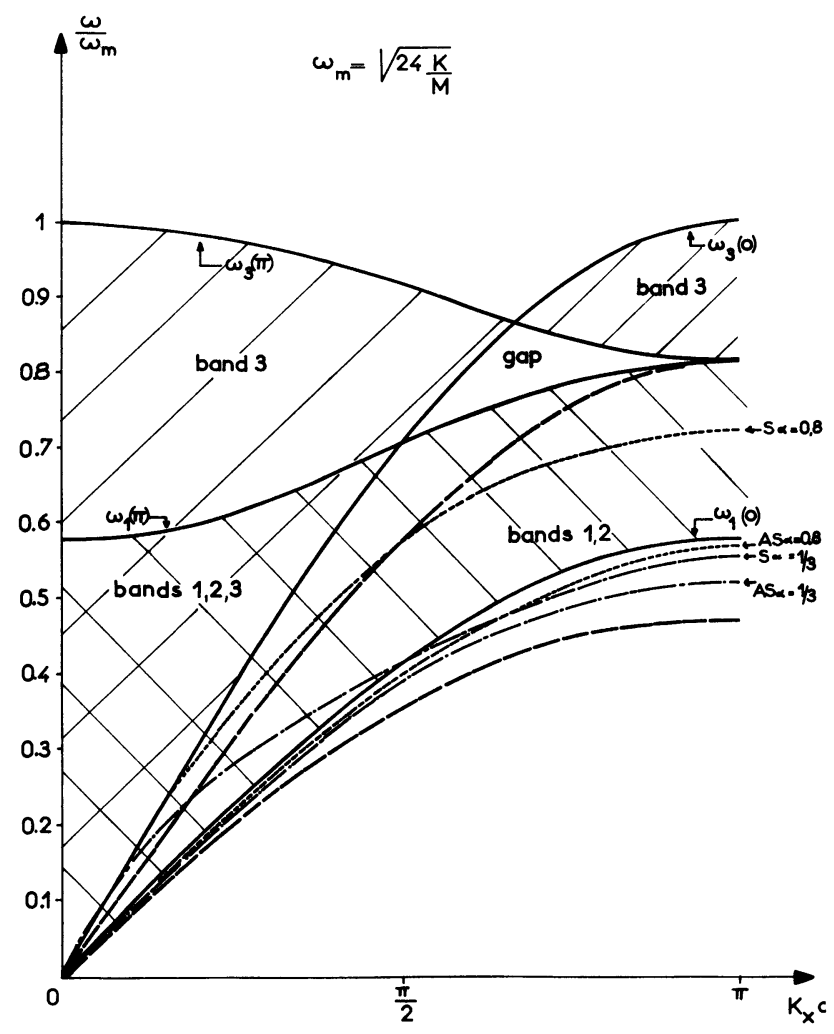

FIG. 1. - Localized and resonant phonons at a planar defect for $\alpha=K^{\prime} / K=\frac{1}{3}$ and 0.8 . The hachured regions are the bulk bands. In - - lines are given the free surface localized and resonant phonons ; in - - - - - - - and . - lines the localized and resonant phonons for the planar defect respectively for $K^{\prime} / K=0.8$ and $\frac{1}{3}$.

(*) A. Blandin, private communication. the mirror plane. All the physical information we need is contained in the determinant

$$
\Delta=|I-V G|
$$

where $V$ is the perturbation of the dynamical matrix due to the planar defect and $G$ is the bulk Green's function [10]. The block diagonalization described above enables us to treat separately the symmetric and antisymmetric part of $\Delta$. Each of these determinants is $(3 \times 3)$, due to the three directions of polarization $(x, y, z)$, and to the fact that the perturbation is localized between the planes $n=0$ and $n=1$.

We give the results along the $k_{x}$ axis of the two dimensional Brillouin zone ( $k$ being the propagation vector). In the model used here, the vibrations polarized along $\hat{y}$ decouple from those polarized in the sagittal plane $(\hat{x}, \hat{z})$. It is easy to see that these purely transverse vibrations $(\hat{y})$ give rise to no localized or resonant mode near the stacking fault. We are then left with two $(2 \times 2)$ determinants $\Delta_{\mathrm{S}}$ and $\Delta_{\mathrm{AS}}$ to be solved for the vibrations corresponding to vibrations polarized in the sagittal plane. Let us note at once that in the $\hat{z}$ direction the symmetric displacements dilatate the planar defect and the antisymmetric ones displace it. We therefore expect different frequencies for these symmetric and antisymmetric vibrations.

It is straightforward to write $\Delta_{\mathrm{S}}$ and $\Delta_{\mathrm{AS}}$ for the planar defect, once one knows [10] these determinants for the case $K^{\prime}=0$ (free surfaces) :

$$
\begin{aligned}
\Delta_{\mathbf{S}}\left(\omega^{2}\right)=1 & +\frac{2 K}{M}(1-\alpha) \times \\
& \times\left[(5+\cos \varphi) G_{\mathbf{S}}^{z z}+(1-\cos \varphi) G_{\mathbf{S}}^{x x}\right. \\
& \left.-2 i \sin \varphi G_{\mathbf{S}}^{x z}\right]+16\left(\frac{K}{M}\right)^{2}(1-\alpha)^{2} \\
& \times(1-\cos \varphi)\left[G_{\mathbf{S}}^{x x} G_{\mathbf{S}}^{z z}+\left(G_{\mathbf{S}}^{x z}\right)^{2}\right](1 a) \\
\Delta_{\mathbf{A S}}\left(\omega^{2}\right)=1 & +\frac{2 K}{M}(1-\alpha) \times \\
& \times\left[(1+\cos \varphi) G_{\mathbf{A S}}^{x x}+(1-\cos \varphi) G_{\mathbf{A S}}^{z z}\right. \\
& \left.-2 i \sin \varphi G_{\mathrm{AS}}^{x z}\right]
\end{aligned}
$$

where $M$ is the atomic mass; $\alpha=K^{\prime} / K ; \varphi=k_{x} a_{0}$ ( $a_{0}$ being the lattice parameter). $G_{\mathbf{S}}^{\sigma \sigma^{\prime}}$ and $G_{\mathrm{AS}}^{\sigma \sigma^{\prime}}$ are the symmetric and antisymmetric elements [10] of the bulk Green's function coupling the planes $n=0$ and $n=1$.

In this model we have three bulk bands (see Fig. 1) : whose square frequencies are :

$$
\omega_{1}^{2}\left(k_{z}\right)=\omega_{2}^{2}\left(k_{z}\right)=\frac{4 K}{M}\left(2-\cos k_{x} a_{0}-\cos k_{z} a_{0}\right)
$$

$$
\begin{array}{r}
\omega_{3}\left(k_{z}\right)=\frac{4 K}{M}\left(4-\cos k_{x} a_{0}-\cos k_{z} a_{0}-\right. \\
\left.-2 \cos k_{x} a_{0} \cos k_{z} a_{0}\right) .
\end{array}
$$


Let us first study, eq. (1) in the elastic limit, where our cristal is isotropic by setting

$$
\omega^{2}=\frac{c_{t}^{2}}{a_{0}^{2}} \xi \varphi^{2}
$$

where $c_{\mathrm{t}}$ is the velocity of transverse vibrations.

Below the bulk bands $(\xi<1)$, we expand the defect determinants (1) for small $\varphi$; keeping only the terms necessary to obtain the expansions of the $\omega^{2}$ localized or resonant modes) to the order $\varphi^{4}$ :

$$
\begin{aligned}
& \Delta_{\mathrm{S}}(\xi)=\alpha+\varphi \frac{(1-\alpha)}{\xi}\left\{\left[\left(1-\xi-\frac{\varphi^{2}}{12}\right)^{1 / 2}-\right.\right. \\
& \left.-\frac{3}{2}\left(\frac{3-\xi}{3}\right)^{3 / 2}-\frac{1}{2}\left(\frac{3}{3-\xi}\right)^{1 / 2}+\left(\frac{3-\xi}{3}\right)^{1 / 2}\right] \\
& \left.+\frac{(1-\alpha)}{3}\left[\left(\frac{3}{3-\xi}\right)^{1 / 2}-\left(1-\xi-\frac{\varphi^{2}}{12}\right)^{1 / 2}\right]\right\}+\cdots
\end{aligned}
$$

$$
\Delta_{\mathrm{AS}}(\xi)=\alpha+\frac{\varphi(1-\alpha)}{6 \xi}\left\{4[3(3-\xi)]^{1 / 2}-\right.
$$

$$
\begin{aligned}
& -\frac{3}{\left(1-\xi-\frac{\varphi^{2}}{12}\right)^{1 / 2}}-6\left(1-\xi-\frac{\varphi^{2}}{12}\right)^{1 / 2} \\
& \left.-3\left(1-\xi-\frac{\varphi^{2}}{12}\right)^{3 / 2}\right\}+\cdots
\end{aligned}
$$

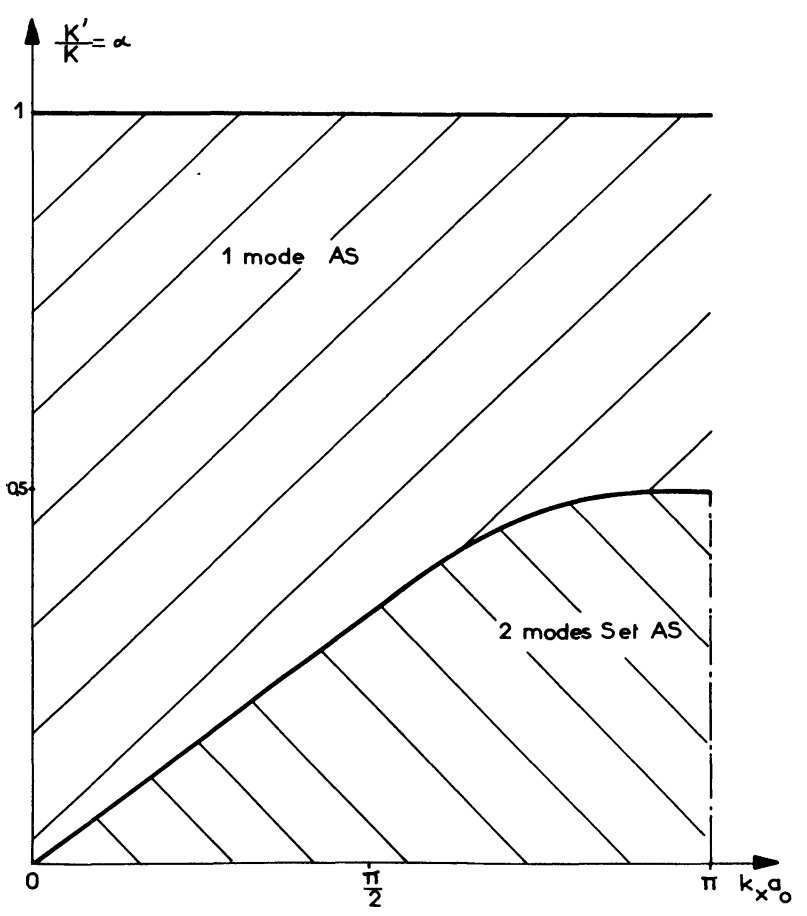

Fig. 2. - Regions of existence of the symmetric and antisymmetric phonons localized near the planar defect.
The localized phonons are obtained for $\Delta=0$. One sees easily that for $\alpha=0$ (free surfaces case) we obtain the well known Rayleigh waves as well from $\Delta_{\mathrm{S}}$ as from $\Delta_{\mathrm{AS}}$. For the planar defect $(\alpha \neq 0)$, these two time degenerate surface modes split into two distinct ones.

By setting $\xi=1$ in ( $4 a$ ) we see that the symmetric localized mode exists only for $\varphi>\varphi_{0}$ (see Fig. 2) with

$$
\varphi_{0}=\frac{\alpha}{1-\alpha} \frac{2 \sqrt{6}}{1+2 \alpha} .
$$

Let us note that a symmetric localized mode of the same type was obtained before [7] for a twodimensional defect consisting of an isolated impurity plane bounded by different (than in the bulk) force constants to its two neighbour planes. However, as mentioned previously the model employed in this work [7] does not satisfy the condition of rotational invariance.

By setting $\xi=1-\gamma \varphi^{2}$ in eq. (4b), we obtain

$$
\gamma=\frac{1}{12}+\frac{1}{4}\left(\frac{1-\alpha}{\alpha}\right)^{2}
$$

which gives the following dispersion relation for the AS localized mode :

$$
\omega_{\mathrm{AS}}^{2}=\frac{c_{\mathrm{t}}^{2}}{a_{0}^{2}}\left[1-\frac{\varphi^{2}}{12}-\frac{1}{4}\left(\frac{1-\alpha}{\alpha}\right)^{2} \varphi^{2}\right] \varphi^{2} .
$$

This AS localized mode was of course the only one Kosevich et al. [9] could find in their elastic limit approach. This mode exists for all values of $\alpha<1$ (see Fig. 2).

At $\varphi=\pi$ we obtain the following expressions [13] for the square frequencies of the localized phonons :

$$
\begin{aligned}
& \frac{\omega_{\mathrm{S}}^{2}}{\omega_{\mathrm{M}}}=\frac{2}{9}+\frac{-96 \alpha^{3}+352 \alpha^{2}-408 \alpha+144}{18\left(8 \alpha^{2}-18 \alpha+9\right)(3-2 \alpha)} \alpha \\
& \frac{\omega_{\mathrm{AS}}^{2}}{\omega_{\mathrm{M}}^{2}}=\frac{2}{9}+\frac{-96 \alpha^{3}+272 \alpha^{2}-246 \alpha+72}{18(3-4 \alpha)\left(8 \alpha^{2}-18 \alpha+9\right)} \alpha .
\end{aligned}
$$

- The vibrations associated to these phonons at $\varphi=\pi$ are polarized in the direction normal to the $(001)$ surface.

When there is no coupling of the crystal vibrations between the two parts of crystal $(\alpha=0)$ we obtain $\omega^{2} / \omega_{\mathrm{M}}^{2}=\frac{2}{9}$ which is the square frequency of the free surface modes (S, AS).

For $1<\xi<3$ (in the 1 and 2 bulk bands) we expand also the defect determinants (1) for small $\varphi$ 
keeping only the terms necessary to obtain the $\omega^{2}$ to the order $\varphi^{4}$ :

$$
\begin{aligned}
& \text { Real } \Delta_{\mathrm{S}}=\alpha+\frac{(1-\alpha) \varphi}{\xi} \times \\
& \times\left\{-\frac{3}{2}\left(\frac{3-\xi}{3}\right)^{3 / 2}-\frac{1}{2}\left(\frac{3}{3-\xi-\frac{\varphi^{2}}{4}}\right)^{1 / 2}\right. \\
& \left.+\left(\frac{3-\xi}{3}\right)^{1 / 2}+\frac{(1-\alpha)}{3}\left(\frac{3}{3-\xi-\frac{\varphi^{2}}{4}}\right)^{1 / 2}\right\}+\cdots
\end{aligned}
$$

$\operatorname{Real} \Delta_{\mathrm{AS}}=\alpha+\frac{2}{3} \frac{(1-\alpha)}{\xi} \varphi[3(3-\xi)]^{1 / 2}$.

From $(8 b)$, we see that there is no antisymmetric resonance.

For $\alpha=0$ the equation Real $\Delta_{\mathrm{S}}=0$ gives $\xi=2$ which corresponds to the free surface resonance [10a]. For $\alpha \neq 0$ let :

$$
\xi=3\left(1-A^{2} \varphi^{2}+\cdots\right)
$$

The equation Real $\Delta_{\mathrm{S}}=0$ gives :

$$
A^{2}=\frac{\left[3(1-\alpha)-2(1-\alpha)^{2}\right]^{2}+27 \alpha^{2}}{(18 \alpha)^{2}} \text {. }
$$

This result differs from the result of Kosevich but we can check ours, by noting that this resonance is continued by the symmetric localized mode at $\varphi=\varphi_{0}$ (eq. (5)). One obtains this result by putting $\xi=1$ in $(8 a)$. This resonance

$$
\omega_{\mathrm{R}}^{2}=3 \frac{c_{\mathrm{t}}^{2}}{a_{0}^{2}}\left(1-A^{2} \varphi^{2}\right) \varphi^{2}
$$

is well defined [10] if

$$
\Gamma=\left(\operatorname{Im} \Delta_{\mathrm{S}}\right) / \frac{\partial\left(\operatorname{Real} \Delta_{\mathrm{S}}\right)}{\partial \omega^{2}} \ll \omega_{\mathrm{R}}^{2} .
$$

For $\varphi \ll \varphi_{0}$, we found

$$
\frac{\Gamma}{\omega_{\mathrm{R}}^{2}}=\frac{2 \sqrt{2}}{81 \sqrt{3}} \frac{(1-\alpha)^{5}(1-2 \alpha)}{\alpha^{3}} \varphi^{3}
$$

and in this limit, the resonance is found to be a sharp peak in the bulk density of states.

On figure 2, we give along the $k_{x}$ axis, the dispersion curves obtained from eq. (1) for the localized resonant modes. The results for $\alpha=\frac{1}{3}$ is given to illustrate a case where the $S$ mode is resonant in the elastic limit and localized outside. In the $\alpha=0.8$ case the $S$ branch is completely inside the bulk bands and the AS branch is just below the bulk bands. One expects [1] in general the force constants near stacking faults to be almost the same as in the bulk. However, phenomenas of the type described here should exist (at least for the long wavelength phonons) at highly desoriented grain boundaries, if they are not partially coherent.

This study is now extended [12] to the case of an interface between two different media and to the case of interface superstructures.

Acknowledgements. - We would like to thank Pr. J. Friedel for helpful comments.

\section{References}

[1] Friedel, J, in Dislocations (Pergamon Press) 1964.

[2] See for references : LenglaRT, P., DobrzYNSKI, L. et LEMAN, G., Ann. Phys. 7 (1972) 407 and Maradudin, A. A. Montroll, E. W., Weiss, G. H. and Ipatova, I. P. (Academic, New York) 1971.

[3] LudwiG, W., Springer Tracts Mod. Phys. 43 (1967) 206; LudWIG, W. and LeNGeler, B., Solid State Commun. 2 (1964) 83.

[4] Kobori, I., Progr. Theor. Phys., Japan 33 (1965) 614.

[5] IosilevskiY, Ya. A., Zh. Eksper. Teor. Fiz. 51 (1966) 201 ; Pis'ma Zh. Eksper. Teor. Fiz. 7 (1968) 32; Fiz. Tver. Tela 10 (1968) 961 ; 10 (1968) 1567; Kristallografiya 14 (1969) 577; Fiz. Met. Metalloved. 29 (1970) $735 ; 29$ (1970) 1137 ; 30 (1970) $264 ; 30$ (1970) 701.

[6] Lifshitz, M. and Kosevich, A. M., Report Prog. Phys. 29 (1966) 217.
[7] IosILEVSKIY, Ya. A., Kristallografiya 14 (1969) 201.

[8] IosilevskiY, Ya. A. and Vardanyan, R. A., Fiz. Metallov Metalloved. 33 (1972) 1164.

[9] Kosevich, A. M. and Khokhlov, V. I., Sov. Phys. Solid State 10 (1968) 39.

[10] MASri, P. and Dobrzynski, L., a) J. Physique 32 (1971) 295 ; b) J. Phys. Chem. Solids 34 (1973) 847 ; c) Surf. Sci. 34 (1973) 119.

[11] Blandin, A., Castiel, D. and Dobrzynski, L., Solid State Commun. 13 (1973) 1175

[12] Djafari-Rouhani, B., Masri, P. and Dobrzynski, L., to be published.

[13] Another expression for the results (7) were given in reference $[4 c]$, where a typing error appears : $97 \alpha^{3}$ should read $96 \alpha^{3}$. 